MATEC Web of Conferences 44, 01009 (2016)

DOI: $10.1051 /$ matecconf/20164401009

(C) Owned by the authors, published by EDP Sciences, 2016

\title{
The methodology of multicriterial assessment of Petri nets' apparatus
}

\author{
Dmitry Pashchenko ${ }^{1 a}$, Dmitriy Trokoz ${ }^{2}$, Galina Sovetkina $^{3}$, Ekaterina Nikolaeva ${ }^{4}$, Michael Sinev ${ }^{5}$, Aleksey Dubravin ${ }^{6}$ and \\ Nikolas Konnov ${ }^{7}$ \\ ${ }^{1234567}$ Department of Computer Science, Penza State University, Penza, Russia
}

\begin{abstract}
This article emphasizes the effectiveness and relevance of the using of the apparatus of Petri nets for modeling of complex computing systems. Due to the fact that the methods of analysis existing in this theory do not allow estimating the resources required to build the desired model of the system, there is a problem of shortage of criteria for its evaluation in terms of the complexity of the construction. In the article we consider the method of analysis of a random Petri net based on the complexity of its building and relationships of internal units - subnets. The purpose of this article is a software implementation of such an assessment within the theory of PN structures. Due to the fact, that structural approach allows to perform the operation of decomposition of the original system, this model can be divided into subnets of minimal dimension, that will allow to make its quantitative assessment - ranking. To determine the total assessment of the input and output data of the system we will perform the analysis of head and tail positions of the net taking into account the weights of the input and output arcs of these positions. In order to identify an extent of the cost required to build the system, the number of operations of union of subnet transitions and positions. These subnets have minimal dimension in the original PN. Thus, the article demonstrates the formal implementation of assessment technique modules with using of algebra of sets, and the rules of splitting the PN structure into elementary blocks are formulated. The example of a comparative assessment of the two Petri nets based on the proposed complexity criteria is given; the plots of PN in different coordinate systems are displayed. The article presents the results of the research - a plot of PN structures in three-dimensional space, implemented using described software. It demonstrates the accuracy of PN assessment by structural analysis in comparing with a non-automated visual one. This approach can be applied for comparative assessment of computer systems in terms of complexity of their construction and size of input and output data.
\end{abstract}

\section{Introduction}

The development of modern information technology has already determined the effectiveness of the application of mathematical abstractions in order to identify the properties and behavioral states of complex computing systems. The theories of graphs [1,2], finite automata [3], as well as the apparatus of Petri nets may be the tools of such research. The article will consider the apparatus of Petri nets which has a powerful formal expressiveness in describing and modelling of computing systems.

In addition, works [4,5] show the effectiveness of using of the PN theory as a tool to track the system response to the manifestation of various cause-effect relationships, which are widely used in the model description of parallel processes, in sharing of critical resources, in the search for security, persistence, accessibility and vitality of the system. There are several types of PN: time, scholastic, inhibitory, colored and hierarchical ones. The using of the particular specie of net determines the functionality of the system being modeled. For example, the inhibitory nets $[6,7]$ are used in order to ensure the protection and security of information system, hierarchical ones [8] are to perform the analysis of complex dynamic systems containing embedded nets, scholastic ones [9] are used when it is necessary to provide the random length of the trigging of net transitions.

There are three main groups of methods for analyzing the properties of Petri nets: methods based on the construction of tree of reachable markings and tree of covering markings; matrix methods using the fundamental equation of the net and invariants; reduction methods [10]. It should be noted that the reduction is a complementary research tool and it is a special case of equivalent transformations, reducing the dimension of the net [10].

\section{Statement of the problem}

The mentioned analysis methods do not allow to characterize the system in terms of the complexity and difficulty of its construction.

Let's consider the example in Figure 1.

\footnotetext{
a Corresponding author: dmitry.pashchenko@gmail.com,dmitriy.trokoz@gmail.com, sovetkina-galja@rambler.ru
} 


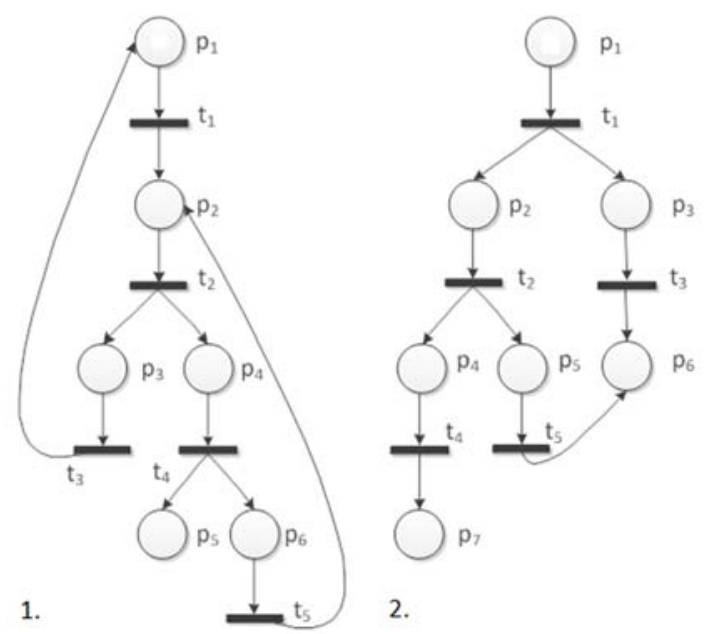

Figure 1. The arbitrary Petri nets.

The nets have the same number of transitions, however, net 1 is composed of 2 cycles, and the network 2 has one position more. It is not possible to determine which of them is more difficult, and with non-automated analysis of bulky computer systems it would be practically impossible.

In order to solve the optimization and verification problems, the concept of structural analysis of system is used; it provides a mechanism of identification of the subsystems of different levels, their relations and connections. Application of the decomposition operation to a discrete system would divide it into several functional parts, which can then be reorganized in order to increase its effectiveness.

Under the grant of RNF for conducting of the fundamental scientific research and exploratory scientific research involving young researchers on the theme "Analysis and synthesis of net structures of complex systems based on tensor and transformational techniques," the task of performing the structural analysis of Petri nets (PN) using the methodology tensor transformations was given. As it is known, tensor calculating $[11,12]$, is widely used in mechanics (elasticity theory), electrodynamics and in the theory of relativity.

During the work it is planned to find numerous alternative variants of primitive $\mathrm{PN}$ by dividing it into partial elements, thereby implementing the conversion of model from one coordinate system to another, using the tensor methodology by introducing space, dimension, defining of the coordinate system, and methods for their conversion. The elementary blocks obtained after decomposition are proposed to be under the operations of union, without breaking the rules of the original system known previously. As a result of the synthesis of the simplest structures we obtain a $\mathrm{PN}$ space of varying degree of equivalency to the original net. For the analysis of the obtained structures we will perform the introduction of the assessment system for the apparatus of Petri nets, given below, in order to select the net with a less complex structure.
Taking into account the mentioned facts, it is planned to implement plotting, that displays a set of synthesized $\mathrm{PN}$ as points in space, using the software.

\section{The formal implementation}

The program will be a set of modules; each of them calculates the index of a particular scale.

\subsection{Module 1. The scale of number of the simplest elements.}

\subsubsection{The input data}

Petri net $P$, described by the generalized matrix.

\subsubsection{Calculating}

Let's divide $P$ into a set of elementary nets

$$
E=\left\{e_{1}, e_{2}, \ldots, e_{\mathrm{k}}\right\}
$$

Elementary net is a net with one input position, one output position and one transition ( Figure 2).

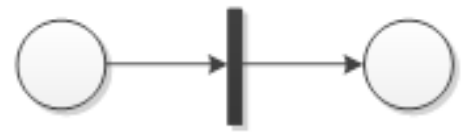

Figure 2. The elementary Petri net.

First we hold the decomposition of the net into linear base fragments (LBF) - cycles and chain sequences of transitions and positions, and we get the simplest ones of them. Splitting into linear base fragments (LBF) will be produced with using of the following rules:

1) We perform the splitting of transitions, which have more than one input or output position. The original net is given in the Figure 3 (a), and the net with divided transitions $t_{1}$ and $t_{3}$ is given in the Figure $3(\mathrm{~b})$.

2) We exclude the cycles from the obtained net (if there are cycles in the net), dividing positions and transitions if it is necessary, without breaking of existing links. A net element (transition or position) will be divided in case when it has more than one output element or input element (Figure 3c).

3) We isolate the linear sequences of transitions, performing the dividing of positions, if it is necessary, as in the previous step (Figure 3d). 


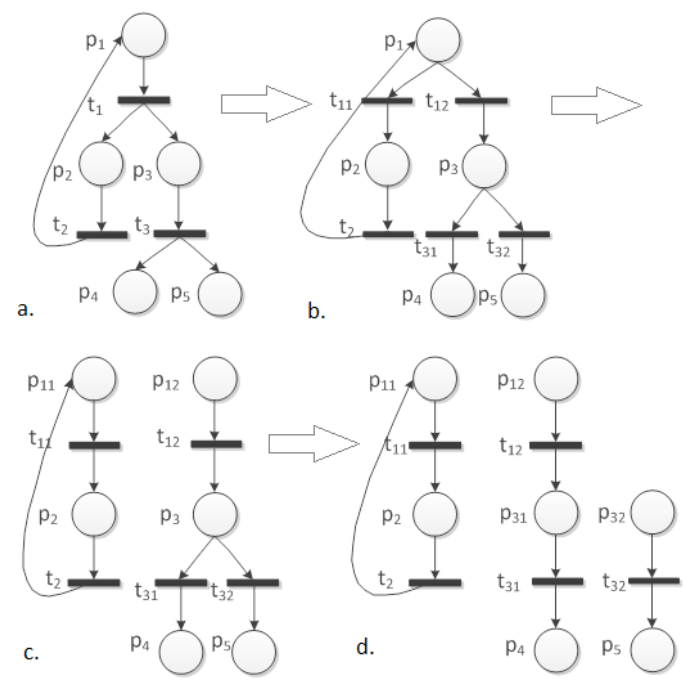

Figure 3. The example of dividing PN into LBF.

To formalize the process of dividing into elementary blocks we introduce the operator $\mathrm{J}(P)$, which returns a set of elementary elements of the net P. Thus, the value of the scale will be the power of the resulting set:

$$
f_{1}(P)=|\mathrm{J}(P)|
$$

\subsubsection{The characteristic of system}

It allows defining the rank of the system.

\subsection{The scale of positions' weights}

\subsubsection{The input data}

The incidence matrix $D$ of the net, $s_{1}, s_{2}$ - weights of the head and tail positions, respectively.

\subsubsection{Calculating}

To find the sum of the weights of positions let's use the formula:

$$
f_{2}(D)=s_{1} K_{1}+s_{2} K_{2}
$$

where $K_{1}$ is the number of head positions, $K_{2}$ is the number of tail positions. The values of $K_{1}, K_{2}$ are defined by the incidence matrix. The incidence matrix $D$ has a dimensionality of $m \times n$, where $m$ is a number of positions, $n$ is a number of transitions, and it represents the difference between the input and output matrices:

$$
D=D^{+}-D^{-}
$$

It is clear that element $d_{\mathrm{i}, \mathrm{j}}, 0 \leq i \leq m, 0 \leq j \leq n$ of matrix $D$, determines the ratio of the position and the transition in such a way: $d_{i, j}>0$, if the position belongs to the set of input position of transition, $d_{\mathrm{i}, \mathrm{j}}<0$, if the position belongs to a set of output positions of transition, $d_{\mathrm{i}, \mathrm{j}}=0$ if the position does not belong to any of these sets or belongs to two sets simultaneously, forming a loop. In this formalism we do not take into account the structures of PN which have loops. It follows that for finding of the tail positions of PN, it is necessary to search for such a row of a matrix, in which for $\forall d_{\mathrm{j}}$ the condition $d_{\mathrm{j}} \geq 0$ would be performed. Similarly, for determining of the head position of the structure it is necessary to search for such a row of matrix in which for $\forall d_{\mathrm{j}}$ the condition $d_{\mathrm{j}} \leq$ 0 would be performed.

\subsubsection{The characteristic of system}

The scale allows to make the total assessment of the input and output data that is interesting in terms of the investigation of the behavior of the whole system, and it can be applied to the models of the "black box" type.

\subsection{The scale with the number of operations performing transitions and positions union}

\subsubsection{The input data}

Petri net $\mathrm{P}$ described by the generalized matrix.

\subsubsection{Calculating}

On the stage of the calculation in the module 1, we obtain the sum of the number of dividing of transitions and positions, which in this case will be similar to the sum of the number of union of transitions and positions $f_{3}(P)$.

\subsubsection{The characteristic of system}

It allows to define the complexity of a system.

The assessment of the scales all together allows to analyze such criteria as:

1) The complexity of the overall system. It allows to get the most comprehensive assessment of the complexity of the synthesized PN structure.

2) The effectiveness of parallelization. It allows tracking the degree of parallelism of processes.

3) The assessment of the dimension of the system. The ranking of the system.

To determine the optimal element of discrete set of received net, it is necessary to take into account the original model of the system and tasks that it performs. At this stage, the optimal element of the PN set is the one that has the shortest length of the vector:

$$
R=\sqrt{f_{1}{ }^{2}(P)+f_{2}^{2}(D)+f_{3}^{2}(P)}
$$

Taking into account the available data, a threedimensional space is supposed to be built, but there is the prospect of working with hyperspaces. The result of the work is a plot showing a set of PN as points in space, which allows to allocate the nets with the most simple structure and equivalent to the original network.

\section{An example}

Let's hold the comparative analysis of the complexity of Petri nets presented in Figure 1. For this purpose, we calculate the values for each of them using scales.

\subsection{Scale 1}


The process of dividing of nets into the linear basic fragments is shown in the figures 4 and 5 .

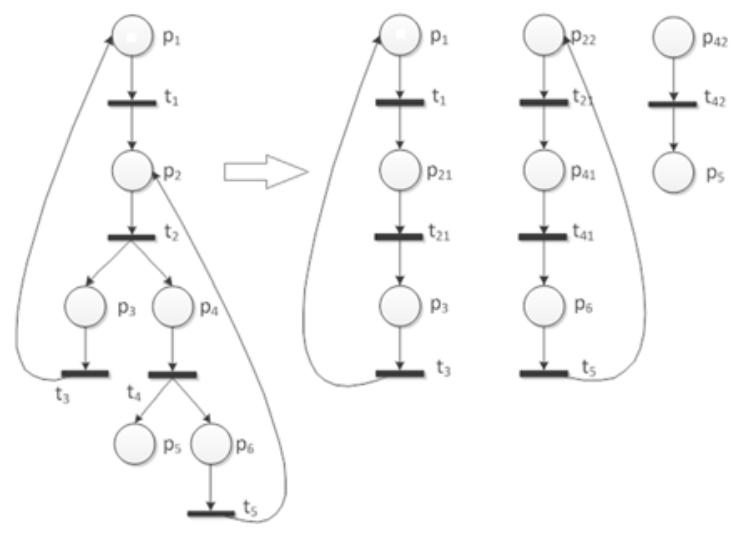

Figure 4. Petri net 1. Dividing into LBF.

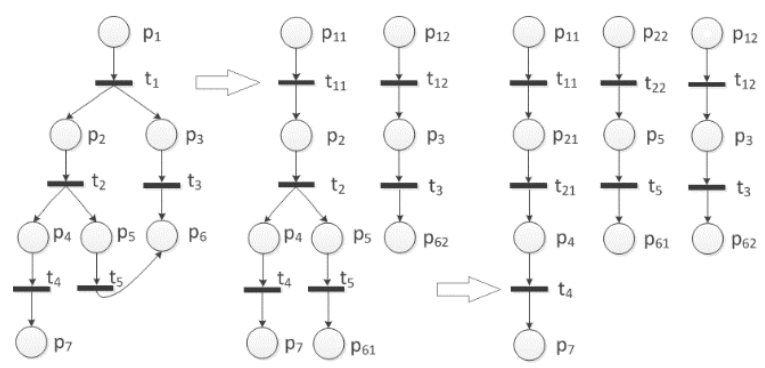

Figure 5. Petri net 2. Dividing into LBF.

We divide the obtained elements into elementary blocks. For the first we obtain a set $E=\left\{e_{1}, e_{2}, \ldots, e_{\mathrm{k}}\right\}$ of elementary Petri nets, each of them is described by a variety of positions and transitions.

Table 1. A set of elementary nets $E_{l}$ for Petri net 1 .

\begin{tabular}{|c|c|c|}
\hline $\begin{array}{c}\text { PN } \\
\boldsymbol{e}_{\mathbf{i}}\end{array}$ & $\begin{array}{c}\text { Set of } \\
\text { positions }\end{array}$ & $\begin{array}{c}\text { Set of } \\
\text { transitions }\end{array}$ \\
\hline$e_{1}$ & $\left\{p_{1}^{\prime}, p_{21}^{\prime}\right\}$ & $\left\{t_{11}\right\}$ \\
\hline$e_{2}$ & $\left\{p^{\prime \prime}{ }_{21}, p_{3}^{\prime}\right\}$ & $\left\{t_{21}\right\}$ \\
\hline$e_{3}$ & $\left\{p^{\prime \prime}{ }_{3}, p_{1}\right\}$ & $\left\{t_{3}\right\}$ \\
\hline$e_{4}$ & $\left\{p_{22}^{\prime}, p_{41}^{\prime}\right\}$ & $\left\{t_{21}\right\}$ \\
\hline$e_{5}$ & $\left\{p^{\prime \prime} 41, p_{6}^{\prime}\right\}$ & $\left\{t_{41}\right\}$ \\
\hline$e_{6}$ & $\left\{p^{\prime \prime}{ }_{6}, p_{22}\right\}$ & $\left\{t_{5}\right\}$ \\
\hline$e_{7}$ & $\left\{p_{42}, p_{5}\right\}$ & $\left\{t_{42}\right\}$ \\
\hline
\end{tabular}

Table 2. A set of elementary nets $E_{2}$ for Petri net 2 .

\begin{tabular}{|c|c|c|}
\hline $\begin{array}{c}\mathbf{P N} \\
\boldsymbol{e}_{\mathbf{i}}\end{array}$ & $\begin{array}{c}\text { Set of } \\
\text { positions }\end{array}$ & $\begin{array}{c}\text { Set of } \\
\text { transitions }\end{array}$ \\
\hline$e_{1}$ & $\left\{p_{11}, p_{21}^{\prime}\right\}$ & $\left\{t_{11}\right\}$ \\
\hline$e_{2}$ & $\left\{p^{\prime \prime}{ }_{21}, p_{4}^{\prime}\right\}$ & $\left\{t_{21}\right\}$ \\
\hline$e_{3}$ & $\left\{p^{\prime \prime}{ }_{4}, p_{7}\right\}$ & $\left\{t_{4}\right\}$ \\
\hline$e_{4}$ & $\left\{p_{22}, p_{5}^{\prime}\right\}$ & $\left\{t_{22}\right\}$ \\
\hline$e_{5}$ & $\left\{p^{\prime \prime}{ }_{5}, p_{61}\right\}$ & $\left\{t_{5}\right\}$ \\
\hline$e_{6}$ & $\left\{p_{12}, p_{3}^{\prime}\right\}$ & $\left\{t_{12}\right\}$ \\
\hline$e_{7}$ & $\left\{p^{\prime \prime}{ }_{3}, p_{62}\right\}$ & $\left\{t_{3}\right\}$ \\
\hline
\end{tabular}

We calculate the capacity of each of obtained sets: $f_{1}\left(P_{1}\right)=\left|\mathrm{J}\left(P_{1}\right)\right|=\left|E_{1}\right|=7, f_{1}\left(P_{2}\right)=\left|\mathrm{J}\left(P_{2}\right)\right|=\left|E_{2}\right|=7$.
From these calculations, it follows that for the construction of both net, the same number of blocks is required.

\subsection{Scale 2}

$$
f_{2}(D)=s_{1}+K_{1}+s_{2} K_{2}
$$

We take the coefficients s1 and s2 equaling 1 for both nets.

Let's find the quantity of head and tail positions of net 1 , using the incidence matrix $D_{1}$.

Table 3. The incidence matrix $D_{1}$.

\begin{tabular}{|c|c|c|c|c|c|}
\hline & $t_{1}$ & $t_{2}$ & $t_{3}$ & $t_{4}$ & $t_{5}$ \\
\hline$p_{1}$ & 1 & 0 & -1 & 0 & 0 \\
\hline$p_{2}$ & -1 & 1 & 0 & 0 & -1 \\
\hline$p_{3}$ & 0 & -1 & 1 & 0 & 0 \\
\hline$p_{4}$ & 0 & -1 & 0 & 1 & 0 \\
\hline$p_{5}$ & 0 & 0 & 0 & -1 & 0 \\
\hline$p_{6}$ & 0 & 0 & 0 & -1 & 1 \\
\hline
\end{tabular}

Table 4. The incidence matrix $D_{2}$.

\begin{tabular}{|c|c|c|c|c|c|}
\hline & $t_{1}$ & $t_{2}$ & $t_{3}$ & $t_{4}$ & $t_{5}$ \\
\hline$p_{1}$ & 1 & 0 & 0 & 0 & 0 \\
\hline$p_{2}$ & -1 & 1 & 0 & 0 & 0 \\
\hline$p_{3}$ & -1 & 0 & 1 & 0 & 0 \\
\hline$p_{4}$ & 0 & -1 & 0 & 1 & 0 \\
\hline$p_{5}$ & 0 & -1 & 0 & 0 & 1 \\
\hline$p_{6}$ & 0 & 0 & -1 & 0 & 1 \\
\hline$p_{7}$ & 0 & 0 & 0 & -1 & 0 \\
\hline
\end{tabular}

$$
K_{1}=1, K_{2}=1, f_{2}\left(D_{2}\right)=2 .
$$

From the obtained values it can be concluded that the Petri net 2 exceeds Petri net 1 in the total amount of input and output data.

\subsection{Scale 3}

Online references will be linked to their original source, only if possible. To enable this linking extra care should be taken when preparing reference lists.

For calculating the value on the scale it is necessary to know how many unions of positions and transitions of elementary nets we need to make to get the original Petri net. Obviously, the number of unions is identical to the number of dividing of positions and transitions. Let's find such positions and transitions for the first net:

$p_{1}=p_{1}^{\prime}+p^{\prime \prime}{ }_{1}$

$p_{2}=p_{21}+p_{22}$

$p_{21}=p_{21}^{\prime}+p_{21}^{\prime \prime}$

$p_{22}=p_{22}^{\prime}+p_{22}^{\prime \prime}$

$p_{3}=p_{3}^{\prime}+p_{3}^{\prime \prime}$

$p_{4}=p_{41}+p_{42}$

$p_{41}=p_{41}^{\prime}+p_{41}^{\prime \prime}$

$p_{6}=p_{6}^{\prime}+p_{6}^{\prime \prime}$

$t_{2}=t_{21}+t_{22}$

$t_{4}=t_{41}+t_{42}$

We have $f_{3}\left(P_{1}\right)=10$. We make the similar equations for the second net:

$p_{1}=p_{11}+p_{12}$

$p_{2}=p_{21}+p_{22}$ 
$p_{21}=p_{21}^{\prime}+p_{21}^{\prime \prime}$

$p_{3}=p_{3}^{\prime}+p_{3}^{\prime \prime}$

$p_{4}=p_{4}^{\prime}+p^{\prime \prime}{ }_{4}$

$p_{5}=p^{\prime}{ }_{5}+p^{\prime \prime}{ }_{5}$

$p_{6}=p_{61}+p_{62}$

$t_{1}=t_{11}+t_{12}$

$t_{2}=t_{21}+t_{22}$

We have $f_{3}\left(P_{2}\right)=9$. higher.

The conclusion: the complexity of net 1 building is

The following values were obtained as results of calculations:

Table 5. Results of calculations.

\begin{tabular}{|c|c|c|c|}
\hline & $f_{1}$ & $f_{2}$ & $f_{3}$ \\
\hline$P_{1}$ & 7 & 1 & 10 \\
\hline$P_{2}$ & 7 & 2 & 9 \\
\hline
\end{tabular}

The assessment of PN structures in different coordinate systems is given below. Figure 6 shows the dependence of a structure rank of the total weights of the input and output data, Figure $6 \mathrm{~b}$ shows the dependence of the structure rank of the number of operations of transitions and positions union in the elementary PN. After analyzing the comparative characteristics charts, we can conclude that the system described by PN 1, operates with a smaller total amount of input and output data than PN 2. A system described by PN 2, requires less work intensity for its construction.

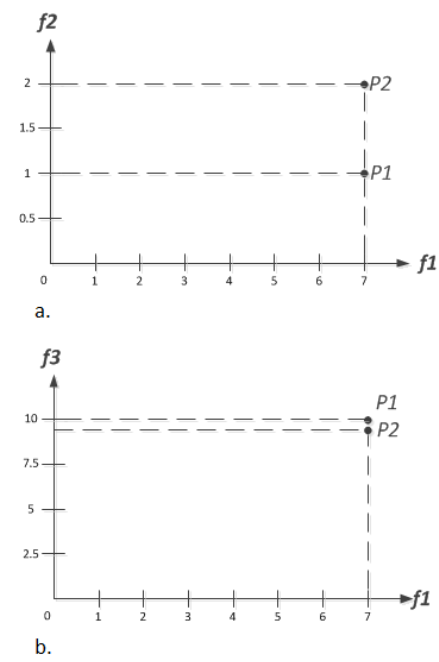

Figure 6. PN in different systems of coordinates.

For the convenience of the visual analysis of structures let's combine axes $f_{1}$ and get a chart of the comparative characteristics in three-dimensional space, shown in Figure 7.

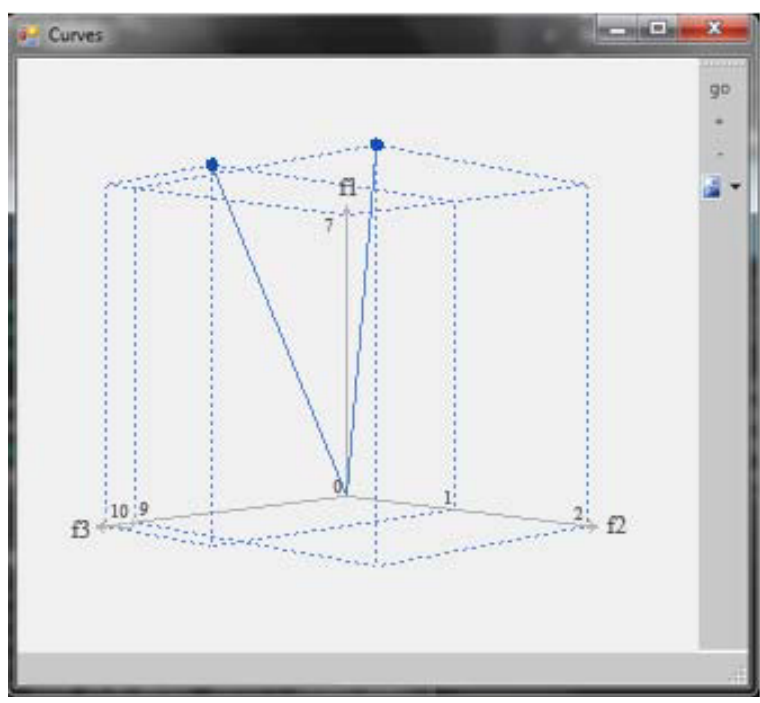

Figure 7. Displaying of $\mathrm{PN}$ in three-dimensional space.

This graph is the result of the program described above, which performs a calculating of values for each Petri net using scales and graphically displays the received values as points in three-dimensional space.

Let's calculate the length of the obtained vectors:

$\left|\overline{P_{1}}\right| \approx 12,2,\left|\overline{P_{2}}\right|=11,5$.

$\left|\overline{P_{1}}\right|>\left|\overline{P_{2}}\right|$, then the first net is more complex than the second one, taking into account the total assessment in all the criteria of complexity.

\section{Conclusion}

A formalized methodology for quantifying assessment of the complexity of arbitrary Petri nets is described.

During the further research it is planned to develop an algorithm that determines the effectiveness of the assessment system for structures and the extent of the need to introduce new scales.

\section{Acknowledgements}

This work was performed as part of the RNF grant for conducting the fundamental scientific research and exploratory scientific research involving young researchers on the theme "Analysis and synthesis of complex net structures of complex systems based on tensor and transformational methods" (convention № 1511-10010).

\section{References}

1. V.P. Kulagin, Automatic Control and Computer Sciences, 23, 55-61, (New York, 1989)

2. B. Bollobas, Graph theory: an introductory course, 63, (2012)

3. H. Straubing, Finite automata, formal logic, and circuit complexity, (2012)

4. T. Agerwala, Computer, 12, 85-94, (1979)

5. T. Agerwala, M. Flynn, ACM SIGARCH Computer Architecture News, 2(4), 81-86, (1973) 
6. D. Pashchenko, D. Trokoz, N. Konnov, and M. Sinev, Procedia Computer Science, 2015, 99-103, (2015)

7. D.A. Zaitsev. Systems Research and Information Technologies, 2, 26-41, (2012)

8. K. Jensen, G. Rozenberg, (Eds.), High-level Petri nets: theory and application, (2012).

9. F. Tüysüz, C. Kahraman, Expert Systems with Applications, 37,5, (2010)

10. D.A. Zaitsev, Cybernetics and Systems Analysis, 42, 1, 126-136, (2006)

11. W. Hackbusch, Tensor spaces and numerical tensor calculus, 42, (2012)

12. A.J. McConnell, Applications of tensor analysis, (2014) 